\title{
The interviewing computer: A technology for gathering comprehensive treatment information
}

\author{
HUGH V. ANGLE \\ WESTAT, 1650 Research Boulevard, Rockville, Maryland 20850
}

\begin{abstract}
The interviewing computer is a practical device for gathering comprehensive information on mental health patients. The complex decisions of a treatment science will require a large body of patient facts and an automated interviewing system to elicit such facts, covering the patient's intake assessment, therapeutic progress, and follow-up stages of treatment. Evidence suggests that the computer interview may equal or excel the interview accomplishments of some clinicians who are unknowledgeable about asking certain clinical questions, forget to ask these questions, or fail to record crucial patient facts. The documentation of each patient's treatment episode is essential for a treatment science, and the computer interview may represent a technology for achieving this information goal.
\end{abstract}

Patient assessment information has two important roles in mental health treatment. First, this information is required by the therapist to formulate treatment decisions. Second, patient information, along with treatment outcome results, determines whether the treatment decisions are appropriate and whether more effective ones are needed. The failure to acquire patient information diminishes the therapist's capacity to plan a course of treatment and impedes the mental health field in developing a science of treatment.

A mental health treatment science has not progressed as rapidly as some clinical investigators would have hoped. Many patients do not benefit from treatment, and this fact has precipitated arguments of whether treatment, or, specifically, psychotherapy, is of great value (Eysenck, 1952) and has raised a controversy over the proportion of patients that treatment helps (Bergin, 1971). Unfortunately, the important issue of the treatment controversy remains clouded. The inability of the mental health field to specify why patient care succeeds or fails indicates a lack of information to resolve this controversy. Adequate and sufficient patient-treatment information would help shed light on this problem.

The difficulties of gathering patient information are due partly to the complexities of the treatment process and partly to the inefficiencies of current data collection procedures. The purpose of this article is to describe a data collection procedure that is more efficient than those currently in use. This description involves the information gathering capabilities of the on-line interactive computer designed to interview mental health patients. The interviewing computer can be utilized to collect three categories of clinical information that are essential in formulating and validating treatment decisions: patient assessment, treatment progress, and outcome information.

\section{ASSESSMENT INFORMATION}

The clinical information associated with mental health treatment is largely the product of the human interview. For many patients, their plan of care is based almost entirely on self-report interview material. An interactive computer, programmed to present multiplechoice questions via a CRT video/typewriter device, can gather patient self-report data. A computer system designed to model the intake assessment of human interviewers could be a useful aid, especially if human performance of this task was defective or inefficient. An automated interview system, however, must be justified in regard to performing a function that most clinicians easily manage on their own and claim to perform successfully.

One way to compare the computer and human interviews is to subject the same patient to the two procedures of intake assessment and judge whether both obtain the same results. If the computer and human interviewer gather the same information, then the computer's justification may only amount to saving the clinician time and effort. On the other hand, if the computer obtains more information than the clinician and, furthermore, elicits certain critical information that the clinician failed to report, then the computer may have an essential clinical function independent of its timeand labor-saving features.

In a mental health center, Angle, Johnsen, Grebenkemper, and Ellinwood (1979) compared the evaluation of patients interviewed by both clinicians and a computer. The computer interview was found to yield considerably more general and specific detail than the clinicians. Moreover, the clinicians failed to identify, or at least to document, $76 \%$ of 20 critical problem items that a group of 55 patients revealed to computer inter- 
rogation and that experts had previously agreed were important patient findings. Angle, Ellinwood, and Carroll (1978) reported a similar clinician-computer reliability study that involved greater experimental controls. Four clinicians interviewed the same four patients, who were also interviewed by the computer. The clinicians' interviews were tape-recorded, transcribed verbatim, and evaluated by raters. The interreliability, or interviewer consistency of identifying similar problems in the same patients, was extremely poor, and the agreement between human interviewers and the computer interview was of a similar magnitude.

The computer-human reliability studies suggest that the human clinical interview has serious reliability (consistency) problems. While the studies do not disclose the relationship between interinterviewer reliability and treatment outcome, the evidence suggests that the treatment of a given patient must logically vary as a function of who performs the interview.

Interview consistency is a strong and attractive feature of computer interviewing. Two computers with the same interview material produce identical results, and two patients taking the same computer interview are presented the same questions, provided they answer alike (computer branching precludes different patients' receiving the same computer material). Formal information structure, consisting of explicit information content and rules governing the presentation of this content (branching), is essential for programming the interviewing computer. For most human interviewers, this structure is generally boring, tedious, and antithetical to the individual approach to interview content. When clinicians are given structured interview guides to follow, they often fail to ask every question or to record every answer (Spitzer \& Endicott, 1974). It is hardly surprising that the computer interview system, programmed to consistently present and record interview material, is more reliable or consistent than some human interviewers are.

Another advantage of the computer interview is that it can provide a more thorough intake assessment than many clinicians can. In the above reliability studies, the thoroughness of the particular computer system was due mainly to the fact that the interview contained a vast number of questions. At present, our computer intake interview consists of over 3,500 questions that include 29 life-problem areas. The development of this system explains why it may surpass the thoroughness of many clinicians. In the beginning of this project, the computer interview consisted of a few hundred items that resulted in a brief report or computer printout. Patients were interviewed by both the computer and clinicians, and the clinicians were systematically asked to describe what more they had discovered from their personal interviews than from the computer printouts. The discovered information was added to the computer so that future computer patients would receive a more thorough interview.
The clinician's input was only one procedure employed to establish the content validity of the computer interview; however, this input contributed an enormous variety of content to the computer interview. The clinician's input represented a bootstrap development of the system in which the computer was programmed to approach the information level of a skilled clinician. An advantage of this method is that the computer program can utilize information from a set of experts that is larger and more diverse than most clinicians can command on their own.

The thoroughness of the computer interview has meant, however, a lengthy 4- to 8-h patient interview and has produced a detailed computer printout that is overwhelming to many clinicians, even though patient responses that are not judged to be problems are not printed. Clinicians have criticized the computer interview because of its length and detailed patient description. On one hand, these criticisms are deserved by the fact that the patient data are of little value until they can be reduced to manageable facts useful to the practicing clinician. On the other hand, those individuals objecting to the scope and depth of a comprehensive interview have not offered realistic alternatives. The hope of discovering a few key patient variables that convey all there is to clinically know about the patient and that suffice to indicate the prescribed patient treatment has not been realized. Patient typologies and psychiatric diagnoses are examples of this approach.

An alternative approach consists of gathering comprehensive information that represents a vast range of patient facts. The full weight of this information is applied to the task of prediction. Clearly, not every fact will contribute to the prediction of a particular outcome, nor will many contribute to any significant prediction. However, our present knowledge of forecasting patient outcomes provides scant basis to prejudge the merits of most clinical information. Therefore, in the early development of the comprehensive interview, the system will contain information of varying clinical quality.

Neither the approach of discovering a few key patient variables or that of gathering comprehensive patient information can be justified by proven accomplishments. The first approach has had a longer history and models the theory-based procedure of physical science; that is, a theory serves to direct and focus data gathering activities. The second approach is a throwback to that of the early empiricists who applied the inductive method almost to the exclusion of theory. A purely inductive approach has few proponents in the clinical field due to the complexity of human behavior and to the lack of an instrument to survey this complexity. The few investigators who are challenged by this complexity and who resist simplifying assumptions about human behavior are usually discouraged by the huge magnitude of the assessment task. For example, Loehlin (1968) claims that any method based on a few patient scores will not 
achieve prediction beyond modest success, and he argues that good patient prediction will require thousands of facts about the patient and the patient's situation. Loehlin has questioned, however, whether the collection of massive information on each patient is feasible.

The computer interview can assess the complexity of patient behavior, but, to date, little progress has been made in this direction. Angle and Ellinwood (1978) argue that the extent and depth of the computer interview must be enlarged to include treatment process and outcome information, as well as other information categories of intake assessment, before any worthwhile reduction of this information can occur. The arguments for a lengthy interview are based, first, on the immense range of variables that influence treatment and, second, on the complexity of treatment decisions.

The range of treatment influences has been indicated by McLean (1974) and Paul (1967), who have both outlined similar lists of factors affecting a patient's treatment (see Table 1). These lists describe broad categories of information. However, each category represents a large number of specific variables. The patient's assessment must deal at the level of specific variables rather than broad categories. This level of assessment will accommodate the complexity of mental health treatment decisions. The complexity of these decisions is described by Bergin (1971), Kazdin and Wilson (1978), and Paul (1967) as the discovery of what treatment, by whom, for this individual, with that problem, under which circumstance is most effective. Each factor is an involved set of decisions, and the factors together represent a large multidimensional approach to a highly complex prediction task. The task is unlikely to be accomplished with a few global predictor variables; and, more important, the discovery is dependent on a data gathering procedure that is more efficient than are those currently utilized by most clinicians and researchers.

Table 1

Variables Influencing Treatment Outcome

\begin{tabular}{|c|c|}
\hline Paul (1967) & $\begin{array}{c}\text { McLean (1974) } \\
\text {-Examples }\end{array}$ \\
\hline $\begin{array}{l}\text { 1. Patient } \\
\text { Distressing behaviors } \\
\text { Personal-social characteristics } \\
\text { Phy sical-social environment } \\
\text { 2. Therapist } \\
\text { Therapeutic technique } \\
\text { Personal-social characteristics } \\
\text { Physical-social environment } \\
\text { 3. Time } \\
\text { Initial contact } \\
\text { Pretreatment } \\
\text { Initial treatment } \\
\text { Main treatment } \\
\text { Termination } \\
\text { Posttreatment }\end{array}$ & $\begin{array}{l}\text { 1. Patient } \\
\text {-sex } \\
\text {-age } \\
\text { 2. Psychological } \\
\text {-expectancies } \\
\text {-motivation } \\
\text { 3. Problems } \\
\text {-self-control } \\
\text {-problem history } \\
\text { 4. Treatment } \\
\text {-approach } \\
\text {-duration } \\
\text { 5. Therapist } \\
\text {-sex } \\
\text {--skills } \\
\text { 6. Environment } \\
\text {-friends }\end{array}$ \\
\hline
\end{tabular}

The computer interview system described by Angle et al. (1978) is extensive, but even so, it lacks many of the information categories outlined in Table 1. Moreover, while the interview covers a wide range of lifeproblem areas, it is basically an intake screen. Angle et al. view this screen as lacking interview depth, in spite of its large question set. These investigators have proposed a series of second-pass surveys or in-depth computer interviews in which an in-depth survey would be associated with each life-problem area. To date, with the exception of an in-depth sexual survey and an in-depth obesity survey, the majority of second-pass interviews have not been developed.

\section{TREATMENT PROGRESS INFORMATION}

Once treatment begins, the therapist requires ongoing information to reveal the progress of treatment. This information informs the therapist whether to continue the present treatment approach, to modify this approach, or to seek a new one in light of unsatisfactory patient progress. The human interview has been the main source of treatment feedback for the therapist. Yet, it is doubtful that human inquiry of patient progress is immune to the same human reliability deficiencies as those observed with the human interview during intake assessment.

The computer interview can be a more systematic procedure for answering the questions of treatment progress. Angle et al. (1978) describe the operation of their computer interview system as a procedure to measure treatment progress. Following intake assessment in which the patient completes the entire computer interview, the therapist selects those life-problem areas (e.g., depression, nonassertion, marriage) on which treatment is being focused. The computer interview is programmed to present the therapist-selected areas repeatedly throughout treatment, and, of course, problem areas can be added or deleted as treatment evolves. The presentation of two or three areas, for example, takes a patient approximately 15 to $45 \mathrm{~min}$ to complete and could be scheduled before the start of every second or fourth treatment session. Angle et al. (1979) describe and illustrate a patient's computerized progress report in which specific problems were summarized into problem-oriented record (POR) categories and standardized with respect to a psychiatric population. The differences between pre- and post-POR category scores were reported as gain scores for a 6-month period.

The therapist's interaction with the patient represents an important information component of treatment. This component has two categories of information: the therapist treatment activities (process information) and the therapist characteristics, such as professional experience, orientation, attitudes, and attributes that bear upon treatment (e.g., sex, age, race). The computer interview can be programmed to gather both types of therapist information. For example, at the end of every treatment 
session, a minimum set of process questions can be presented. A set of 20 to 40 process questions, mostly relating to the life areas selected by the therapist as treatment targets, would take the therapist no longer than 2 to 6 min to answer with a CRT device.

The information about therapist characteristics may be gathered in the same automated manner as the information about patient characteristics. In this instance, the computer instrument is a therapist interview. Each therapist would initially receive an intake computer interview and then receive periodic assessments to survey therapist characteristics that are likely to change as the result of experience and training.

\section{TREATMENT OUTCOME INFORMATION}

The collection of treatment outcome or follow-up data supplies an essential information component for developing a treatment science. The importance of outcome data is illustrated by the simplified decision formula $d / x=y$, in which, given information $x$ about a particular patient, the following treatment decision $d$ will have a probable outcome $y$. In the mental health field, one side of the decision equation is effectively an unknown quantity: knowledge of treatment outcome. In comparison with other types of typically surveyed mental health information, treatment outcome information is poorly represented. In contrast, the opportunity for gathering outcome information is substantial, based on the vast number of patients annually receiving mental health treatment. It must be asked why only an insignificant proportion of these patients are ever evaluated following their treatment episode; the answer may establish the major justification for adopting the computer interview system.

Ideally, every person receiving treatment should also receive a detailed follow-up evaluation. Systematic collection of this information could help resolve the treatment controversy about patient care efficacy. The problem, however, is that no one takes responsibility for follow-up evaluation. Practicing clinicians have traditionally resisted the notion of conducting follow-up evaluations, arguing that it is a laborious task and they cannot spare the time. Program evaluators would gather the data themselves, but they do not have direct or easy access to patients and must rely on the clinician for this contact.

Regardless of who is responsible for conducting outcome evaluation, the fact remains that the failure to systematically gather treatment outcome data is one of the most serious hindrances to creating a science of treatment. One reason the problem continues without resolution is that little has been done to ease the laborand time-consuming features of this task. Indeed, there is a growing pessimism within the mental health field that the task may be impossible. Zusman and Ross (1969) claim that outcome studies will never become a routine part of quality evaluation of individual treatment facilities. They view outcome investigation as representing expensive techniques of special research projects, and they predict that many years will pass before outcome studies are accepted in treatment programs, even on a limited basis.

Zusman and Ross (1969) did not envision the solutions afforded by computer interview technology when commenting on the future of outcome studies, but, since then, a number of investigators have proposed the on-line computer as a means to gather treatment outcome data (Angle \& Ellinwood, 1978; Johnson, Giannetti, \& Williams, 1976; Klein, Greist, \& VanCura, 1975; Stillman, Roth, Colby, \& Rosenbaum, 1969). The on-line computer has the potential of making outcome evaluation a routine activity of a treatment program. The advantage of the computer interview is that it can minimize many research difficulties that make outcome investigation a laborious task for research workers, such as collecting, coding, verifying, and entering into a computer patient outcome data. The operation of the computer interview represents a direct data entry procedure: Patient information is automatically stored in the computer without human intervention and is available for immediate analysis. Moreover, the computer, rather than the data technician, provides editing and some correction of the data. Unlike the research worker, the interviewing computer is available $24 \mathrm{~h} /$ day, 7 days/week. One multiuser computer can handle a large number of data entry terminals, and the terminals need not be in close proximity to the computer. Progress in the field of telecommunications and significant cost reduction in computer hardware will someday allow the patient's home environment to be an origin of data entry.

\section{COMPUTER INTERVIEW AS A PREREQUISITE TO TREATMENT DECISIONS}

The computer interview is a practical procedure of gathering patient self-report material. The computer relieves the therapist of a laborious information task and also provides the therapist a convenient way to record treatment process information. In addition, when the therapist is heavily engaged in treating new patients, the computer interview is readily available to collect outcome data from those patients who have completed treatment. More important, the computer interview ensures detailed, systematic, and documented self-report information. At present, the only other procedure to match the comprehensiveness of the automated interview is the human interview, but the human interview is seldom conducted in a systematic way and seldom yields completely documented information needed to fashion a science of treatment.

The detailed and documented information of the comprehensive computer interview can potentially 
introduce a new era in the investigation of treatment. The collection of assessment, progress, and outcome data on a single patient represents a new approach to treatment investigation. This claim is based on the fact that few, if any, studies have ever attempted to survey detailed information over the entire spectrum of the treatment process. Researchers have divided this process into small manageable parts, studying a few variables at a time. A segmented investigation of a complex process is, however, hardly conducive to a science of treatment. Cronbach (1975) is critical of such investigations because they entail an expectation that results can be synthesized to explain more complex behavior. He suggests that the crucial elements of complex behavior are higher order interactions that are not a part of studies that segment and restrict data collection to narrowly defined aspects of behavior.

A word of caution must be raised to dispel any belief that a treatment science is simply the product of extensive data collection. Accurate treatment decisions will not immediately emerge from a comprehensive computer interview system. A lengthy transition period lies between the collection of massive patient information and the discovery of practical treatment decisions; what is in store during this transition period may discourage many researchers and clinicians.

In the case of clinicians, a comprehensive computer interview presents a huge confusion of patient facts. The clinicians' difficulties with interpreting these facts have been found with computer systems that yield far fewer data than a comprehensive computer interview system. Johnson, Williams, Giannetti, and Schmidt (1977) have observed that clinicians fail to understand how to proceed to decision making based upon the vast collection of computer information. Hedlund, Sletten, Evenson, Altman, and Cho (1977) have commented on the clinician's frustration with not knowing how to use all the computer information provided by their recordkeeping computer system. The difficulty of utilizing clinical information may not be specific to the computer. The computer only sharpens the issue because it documents the problem. How clinicians cope with and assimilate the vast quantity of information from their own interviews has been obscured by the circumstance that human interview material is largely unrecorded or, at best, subject to selective recording.

Mental health researchers will be faced with the major responsibility of translating the comprehensive computer interview information into treatment decisions and with validating these decisions. Previously, researchers have been stymied in this pursuit by the lack of treatment information, especially by the lack of treatment outcome data. While the comprehensive computer interview will improve the availability of patient information, it will also create new problems for the researcher. The researcher may find current psychological methodology, traditionally employed to solve the problem of data complexity, poorly suited to analyzing computer interview data (Angle \& Ellinwood, 1978).

The difficulty that a comprehensive computer inter view system presents to the researcher and, in turn, to a science of treatment is that it is capable of gathering a wide assortment of information on each patient. If programmed to gather comprehensive information per patient, then the interview may not obtain enough information on a sufficient number of patients for worthwhile analysis. The reason for this difficulty is that the scope and depth of the computer interview combine to create a highly abstract individual, that is, a multidimensional computer patient. Moreover, when computer interrogation departs from presenting the same material to all patients (psychological testing) and begins branching (interviewing), each interview branching point differentiates patient subgroups. As the depth of the interview develops, patient subgroups become more numerous and the number of patients per group declines. A data base of 1,000 patients, for example, may encompass only a few individuals, if any, that form a homogeneous group. Unfortunately, the researcher requires more than one or two patients per group for statistical comparison of such groups.

A single-computer system, gathering the comprehensive information of patient assessment, treatment, and outcome, must operate for an almost infinite period to achieve any worthwhile patient population. For this reason, the concept of a computer network is essential to the notion of a comprehensive computer interview system. A network of computer interview systems would accumulate a sizable population of highly abstract computer patients within a reasonable period. A network of similar systems could provide each network user with access to patients with different characteristics and different treatment outcomes under a variety of treatment operations.

Rome (1967) envisioned a large computer network linking many treatment programs as the basis for a quantum advance in psychiatry. The source of Rome's optimism was the substantial improvement in reliability, via standardization, that computers would bring to the clinical field. Improving the reliability of clinical information entails a great achievement but it hardly represents a quantum advance. A marked advance will arise when clinical information is validated, that is, when it is shown to accurately predict the patient's treatment outcome-the basis to formulate treatment decisions. The comprehensive computer interview and computer network processing of interview data can establish the basis for this advance.

\section{REFERENCES}

Angle, H. V., \& Ellinwood, E. H. A psychiatric assessmenttreatment-outcome information system: Evaluation with computer simulation. In F. H. Orthner (Ed.), Proceeding. Second Annual Symposium on Computer Application in Medi- 
cal Care. New York: Institute of Electrical and Electronic Engineers, 1978.

Angle, H. V., Ellinwood, E. H., \& Carroll, J. Computer interview problem assessment of psychiatric patients. In F. $\mathbf{H}$. Orthner (Ed.), Proceeding: Second Annual Symposium on Computer Application in Medical Care. New York: Institute of Electrical and Electronic Engineers, 1978.

Angle, H. V., Johnsen, T., Grebenkemper, N. S., \& Ellinwood, E. H. Computer interview support for clinicians. Professional Psychology, 1979, 10, $49-57$.

BeroIn, A. E. The evaluation of therapeutic outcomes. In A. E. Bergin \& S. L. Garfield (Ed.), Handbook of psychotherapy and behavior change: An empirical analysis. New York: Wiley, 1971.

Cronbach, L. J. Beyond the two disciplines of scientific psychology. American Psychologist, 1975, 30, 116-127.

EysencK, H. J. The effects of psychotherapy: An evaluation. Journal of Consulting Psychology, 1952, 16, 319-324.

Hedlund, J., Sletten, I., Evenson, R., Altman, H., \& Cho, D. W. Automated psychiatric information systems: A critical review of Missouri's Standard System of Psychiatry (SSOP). Journal of Operational Psychiatry, 1977, 8, 5-26.

Johnson, J. H., Giannetti, R. A., \& Williams, T. A. Computers in mental health care delivery: A review of the evaluation toward interventionally relevant on-line processing. Behavior Research Methods \& Instrumentation, 1976, 8, 83-91.

Johnson, J. H., Williams, T. A., Giannetti, R. A., \&
Schmidt, L. J. Strategies for successful introduction of computer technology in a mental health setting: The problems of change. National Computer Conference and Exposition Proceedings, 1977, 46, 55-58.

Kazdin, A. E., \& Wilson, G. T. Evaluation of behavior therapy: Issues, evidence, and research strategies. Cambridge, Mass: Ballinger. 1978.

KleIN, M. H., Greist, J. H., \& VAnCura, L. J. Computers and psychiatry. Archives of General Psychiatry, 1975, 32, 837-843.

LoEHLIN, C. J. Computer model of personality. New York: Random House, 1968.

McLean, P. D. Evaluating community based psychiatric services. In P. O. Davidson, W. Frank, \& L. A. Hamerlynak (Eds.), Evaluation of behavior programs in community, residential, and school settings. Champaign, Ill: Research Press, 1974.

Paul, G. L. Strategy of outcome research in psychotherapy. Journal of Consulting Psychology, 1967, 31, 107-118.

Rome, H. P. Prospects for a Psi-Net: The fourth quantum advance in psychiatry. Comprehensive Psychiatry, 1967, 8, 450-454.

Spitze R, R. L., \& Endicotr, J. Can the computer assist clinicians in diagnosis? American Journal of Psychiatry, 1974, 131, 523-530.

Stillman, R., Roth, W. T., Colby, K. M., \& Rosenbaum, C. P. An on-line computer system for initial psychiatric inventory. American Journal of Psychiatry, 1969, 125, 8-11.

Zusman, J., \& Ross, E. R. Evaluation of the quality of mental health services. Archives of General Psychiatry, 1969, 20, 352-357. 